\title{
Mantel's Theorem for random graphs
}

\author{
B. DeMarco* and J. Kahn ${ }^{\dagger}$
}

\begin{abstract}
For a graph $G$, denote by $t(G)$ (resp. $b(G)$ ) the maximum size of a triangle-free (resp. bipartite) subgraph of $G$. Of course $t(G) \geq b(G)$ for any $G$, and a classic result of Mantel from 1907 (the first case of Turán's Theorem) says that equality holds for complete graphs. A natural question, first considered by Babai, Simonovits and Spencer about 20 years ago is, when (i.e. for what $p=p(n)$ ) is the "ErdösRényi" random graph $G=G(n, p)$ likely to satisfy $t(G)=b(G)$ ? We show that this is true if $p>C n^{-1 / 2} \log ^{1 / 2} n$ for a suitable constant $C$, which is best possible up to the value of $C$.
\end{abstract}

\section{Introduction}

Write $t(G)$ (resp. $b(G)$ ) for the maximum size of a triangle-free (resp. bipartite) subgraph of a graph $G$. Of course $t(G) \geq b(G)$, and Mantel's Theorem [15] (the first case of Turán's Theorem [21]) says that equality holds if $G=K_{n}$. Here we are interested in understanding when equality is likely to hold for the usual ("Erdös-Rényi") random graph $G=G_{n, p}$; that is, for what $p=p(n)$ one has

$$
t\left(G_{n, p}\right)=b\left(G_{n, p}\right) \quad \text { w.h.p. }
$$

(where an event holds with high probability (w.h.p.) if its probability tends to 1 as $n \rightarrow \infty)$. Note that (11) holds for very small $p$, for the silly reason that $G$ is itself likely to be bipartite; but we are really thinking of more interesting values of $p$.

AMS 2010 subject classification: 05D40, 05C35, 05C80

Key words and phrases: Mantel's Theorem, random graph, threshold, max cut

* supported by the U.S. Department of Homeland Security under Grant Award Number 2007-ST-104-000006.

$\dagger$ Supported by NSF grant DMS0701175. 
The problem seems to have first been considered by Babai, Simonovits and Spencer [1], who showed (inter alia) that (11) holds for $p>1 / 2$ (actually for $p>1 / 2-\varepsilon$ for some fixed $\varepsilon>0$ ), and asked whether it could be shown to hold for $p>n^{-c}$ for some fixed positive $c$. This was accomplished by Brightwell, Panagiotou and Steger [2] (with $c=1 / 250$ ), who also suggested that $p>n^{-1 / 2+\varepsilon}$ might be enough. Here we prove the correct result and a little more:

Theorem 1.1. There is a $C$ such that if $p>C n^{-1 / 2} \log ^{1 / 2} n$, then w.h.p. every maximum triangle-free subgraph of $G_{n, p}$ is bipartite.

This is best possible (up to the value of $C$ ), since, as observed in [2], for $p=0.1 n^{-1 / 2} \log ^{1 / 2} n, G_{n, p}$ will usually contain a 5-cycle of edges not lying in triangles. In fact it's not hard to see that the probability in (10) tends to zero for, say, $p \in\left[n^{-1}, 0.1 n^{-1 / 2} \log ^{1 / 2} n\right]$, whereas, as noted above, (11) again holds for very small $p$. An appealing guess is that, for a given $n$, $f(p):=\operatorname{Pr}\left(t\left(G_{n, p}\right)=b\left(G_{n, p}\right)\right)$ has just one local minimum; but we have no idea how a proof of this would go, or even any strong conviction that it's true.

Of course a more general question is, what happens when we replace "triangle" by " $K_{r}$ " (and "bipartite" by " $(r-1)$-partite")? With $t_{r}(G)$ (resp. $\left.b_{r}(G)\right)$ the maximum size of a $K_{r}$-free (resp. $(r-1)$-partite) subgraph of $G$, the natural extension of Theorem 1.1 to general $r$ is

Conjecture 1.2. For any fixed $r$ there is a $C$ such that if

$$
p>C n^{-\frac{2}{r+1}} \log \frac{2}{(r+1)(r-2)} n,
$$

then w.h.p. every maximum $K_{r}$-free subgraph of $G_{n, p}$ is $(r-1)$-partite.

(This is again best possible apart from the value of $C$, basically because for smaller $p$ there are edges not lying in $K_{r}$ 's.) The argument of [2] gives the conclusion of Conjecture 1.2 provided $p>n^{-c_{r}}$ for a sufficiently small $c_{r}$.

The next section states our two main points, Lemmas 2.2 and 2.3, and gives the easy derivation of Theorem 1.1 from these. The lemmas themselves are proved in Sections 4 and 5, following some routine treatment of unlikely events in Section 3, and we close in Section 6 with a few comments on related issues.

Usage. Throughout the paper we use $G$ for $G_{n, p}$ and $V$ for its vertex set. We use $|H|$ for the size, i.e. number of edges, of a graph $H, N_{H}(x)$ for 
the set of neighbors of $x$ in $H$, and $d_{H}(x)$ for the degree, $\left|N_{H}(x)\right|$, of $x$ in $H$. The default value for $H$ is $G$; thus $N(x)=N_{G}(x)$ and, for $B \subseteq V$, $d_{B}(x)=|N(x) \cap B|$ (and $d_{B}(x, y)=|N(x) \cap N(y) \cap B|$ ). For disjoint $S, T \subseteq V$, the set of edges joining $S, T$ in $H$ is denoted $\nabla(S, T)$ if $H=G$ and $H[S, T]$ otherwise.

We will sometimes think of an $R \subseteq\left(\begin{array}{c}V \\ 2\end{array}\right)$ as the graph $(V(R), R)$, with $V(R)$ the set of vertices contained in members of $R$; so for example $N_{R}(x)$ is the set of $R$-neighbors of $x, R[W]$ is the subgraph of $R$ induced by $W \subseteq V$, and " $R$ is bipartite" has the obvious meaning.

When speaking of a cut $\Pi=(A, B)$, we will think of $\Pi$ as either the set of edges $\nabla(A, B)$ or as the ordered partition $A \cup B$ of $V$ (so we distinguish $\Pi=(A, B)$ and $\Pi=(B, A))$. Of course $|\Pi|$ means $|\nabla(A, B)|$.

We use log for $\ln , B(m, p)$ for a random variable with the binomial distribution $\operatorname{Bin}(m, p)$, and " $a=(1 \pm \vartheta) b$ " for " $(1-\vartheta) b \leq a \leq(1+\vartheta) b$." Following common practice, we usually pretend that large numbers are integers, to avoid cluttering the exposition with essentially irrelevant floor and ceiling symbols.

\section{Outline}

We assume from now on that $p>C n^{-1 / 2} \log ^{1 / 2} n$ with $C$ a suitably large constant and $n$ large enough to support the arguments below. In slightly more detail: we fix small positive constants $\varepsilon$ and $\eta$ with $\varepsilon>>\eta$, set $\alpha=.8$, and take $C$ large relative to $\varepsilon$. (The most stringent demand on $C$ is that it be somewhat large compared to $\varepsilon^{-5 / 2}$; see the end of Section 4 . For $\alpha$, any value in $(2 / 3,1)$ would suffice. Apart from this, we will mostly avoid numerical values: no optimization is attempted, and it will be clear in what follows that the constants can be chosen to do what we ask of them.)

Say a cut $(A, B)$ is balanced if $|A|=(1 \pm \eta) n / 2$; though we will sometimes speak more generally, all cuts of actual interest below will be balanced.

We will need the following version of a result of Kohayakawa, Łuczak and Rödl [14]. (See [10, Theorem 8.34] and e.g. [10, Proposition 1.12] for the standard fact that the $G_{n, M}$ statement implies the $G_{n, p}$ version.)

Theorem 2.1. For each $\vartheta>0$ there is a $K$ such that for $p=p(n)>K n^{-1 / 2}$ w.h.p. each triangle-free subgraph of $G=G_{n, p}$ of size at least $|G| / 2$ can be made bipartite by deletion of at most $\vartheta n^{2} p$ edges.

See Section 6 for a little more on Theorem 2.1. 
For a cut $\Pi=(A, B)$, set $X(\Pi)=\left\{x \in A: d_{B}(x)<(1-2 \varepsilon) n p / 4\right\}$ and $T(\Pi)=\left\{x \in A: d_{B}(x)<(1-\varepsilon) n p / 2\right\}(\supseteq X(\Pi))$, and let $Q(\Pi)$ consist of those pairs $\{x, y\}$ from $A$ which either meet $X(\Pi)$ or satisfy one of

(i) $x, y \in A \backslash T(\Pi)$ and $d_{B}(x, y)<\alpha n p^{2} / 2$;

(ii) $|\{x, y\} \cap T(\Pi)|=1$ and $d_{B}(x, y)<\alpha n p^{2} / 4$;

(iii) $\{x, y\} \subseteq T(\Pi)$ and $d_{B}(x, y)<\alpha n p^{2} / 8$.

In addition we set $Q_{v}(\Pi)=\{\{x, y\} \in Q(\Pi):\{x, y\} \cap X(\Pi) \neq 0\}$ and $Q_{e}(\Pi)=Q(\Pi) \backslash Q_{v}(\Pi)$. Note that members of $Q(\Pi)$, while often treated as edges of an auxiliary graph, need not be edges of $G$.

For a cut $\Pi=(A, B)$ and $F \subseteq G$, let

$$
\varphi(F, \Pi)=2|F[A]|+|F[A, B]| .
$$

Lemma 2.2. W.h.p.

$$
\varphi(F, \Pi)<|\Pi|
$$

whenever $\Pi=(A, B)$ is balanced and $F \subseteq G$ is triangle-free with $F \neq \Pi$, $F \cap Q(\Pi)=\emptyset=F[B]$,

$$
|F[A]|<\eta|F[A, B]|,
$$

and

$$
\left|N_{F}(x) \cap B\right| \geq\left|N_{F}(x) \cap A\right| \quad \forall x \in A .
$$

Lemma 2.3. W.h.p.

$$
b(G)>|\Pi|+2|Q|
$$

whenever the balanced cut $\Pi=(A, B)$ and $\emptyset \neq Q \subseteq G \cap Q(\Pi)$ satisfy

$$
d_{Q}(x) \leq d_{B}(x) \quad \forall x \in A .
$$

Given Lemmas 2.2 and 2.3 we finish easily as follows. Let $F_{0}$ be a maximum triangle-free subgraph of $G$, and $\Pi=(A, B)$ a cut maximizing $\left|F_{0}[A, B]\right|$ with (w.l.o.g.) $\left|F_{0}[A]\right| \geq\left|F_{0}[B]\right|$. Since $\Pi$ maximizes $\left|F_{0}[A, B]\right|$, we have (3) (with $F_{0}$ in place of $F$ ) - otherwise we could move some $x$ from $A$ to $B$ to increase $\left|F_{0}[A, B]\right|$-and Theorem 2.1 implies that w.h.p. $F_{0}$ also satisfies (2) (actually with $o(1)$ in place of $\eta$ ). Moreover $\Pi$ is balanced w.h.p., since (w.h.p.)

$$
\begin{aligned}
|\nabla(A, B)| & \geq\left|F_{0}[A, B]\right|>(1-o(1))\left|F_{0}\right| \\
& \geq(1-o(1))|G| / 2>(1-o(1)) n^{2} p / 4
\end{aligned}
$$


and, for example,

$$
|\nabla(A, B)|< \begin{cases}(1+o(1))|A||B| p & \text { if }|A|,|B|>n / 5 \\ (1+o(1)) \min \{|A|,|B|\} n p & \text { otherwise. }\end{cases}
$$

Here the second inequality in (6) is again Theorem 2.1, and the third is the standard observation that $b(G) \geq|G| / 2$ for any $G$. The last inequality in (6) and those in (7) are easy consequences of Chernoff's inequality (Theorem 3.1 below, used via Proposition 3.2 for the second inequality in (7)).

Let $F_{1}=F_{0} \backslash F_{0}[B]$ and $F=F_{1} \backslash Q(\Pi)$. Noting that these modifications introduce no triangles and preserve (2) and (3), we have, w.h.p.,

$$
\begin{aligned}
t(G) & =\left|F_{0}\right| \\
& \leq \varphi\left(F_{1}, \Pi\right) \\
& =\varphi(F, \Pi)+2\left|F_{1} \cap Q(\Pi)\right| \\
& \leq|\Pi|+2\left|F_{1} \cap Q(\Pi)\right| \\
& \leq b(G) .
\end{aligned}
$$

Here (8) is given by Lemma 2.2 and (9) by Lemma 2.3 (the latter applied with $Q=F_{1} \cap Q(\Pi)$ and (5) implied by (3) for $F_{1}$ ).

This gives (11). For the slightly stronger assertion in the theorem, notice that we have strict inequality in (8) unless $F=\Pi$ and in (9) unless $F_{1} \cap$ $Q(\Pi)=\emptyset$. Thus $\left|F_{0}\right|=b(G)$ implies $F_{0}[A]=F[A] \cup\left(F_{1} \cap Q(\Pi)\right)=\emptyset$, so also $F_{0}[B]=\emptyset$ (since we assume $\left.\left|F_{0}[A]\right| \geq\left|F_{0}[B]\right|\right)$.

\section{Preliminaries}

Here we just dispose of some anomalous events. We use Chernoff's inequality in the following form, taken from [10, Theorem 2.1].

Theorem 3.1. For $\xi=B(n, p), \mu=n p$ and any $\lambda \geq 0$,

$$
\begin{aligned}
& \operatorname{Pr}(\xi \geq \mu+\lambda)<\exp \left[-\frac{\lambda^{2}}{2(\mu+\lambda / 3)}\right] \\
& \operatorname{Pr}(\xi \leq \mu-\lambda)<\exp \left[-\frac{\lambda^{2}}{2 \mu}\right] .
\end{aligned}
$$

This easily implies the next two standard facts, whose proofs we omit.

Proposition 3.2. W.h.p. for all $x, y \in V$,

$$
d(x)=(1 \pm o(1)) n p \quad \text { and } \quad d(x, y)=(1 \pm \varepsilon) n p^{2},
$$


Proposition 3.3. There is a $K$ such that w.h.p. for all disjoint $S, T \subseteq V$ of size at least $K p^{-1} \log n$,

$$
|\nabla(S, T)|=(1 \pm \varepsilon)|S||T| p
$$

and

$$
|G[S]|=(1 \pm \varepsilon)\left(\begin{array}{c}
|S| \\
2
\end{array}\right) p .
$$

The next three assertions are also easy consequences of Theorem 3.1 .

Proposition 3.4. There is a $K$ such that w.h.p., for every $\kappa>K p^{-1} \log n$ and $S, T \neq \emptyset$ disjoint subsets of $V$ with $|S| \leq \min \{\kappa,|T|\}$,

$$
|\nabla(S, T)| \leq 2|T| \kappa p
$$

and

$$
|G[S]| \leq|S| \kappa p
$$

Proof. We show (13), omitting the similar proof of (14). For given $s, t$ with $s \leq t$, the number of possibilities for $S$ and $T$ of sizes $s$ and $t$ respectively $(s \leq t)$ is less than $\left(\begin{array}{l}n \\ s\end{array}\right)\left(\begin{array}{l}n \\ t\end{array}\right)<\exp [2 t \log n]$. But for a given $S, T$, since $\mathrm{E}|\nabla(S, T)|=|S||T| p \leq|T| \kappa p$, Theorem 3.1 gives (say)

$$
\operatorname{Pr}(|\nabla(S, T)| \geq 2|T| \kappa p)<\exp [-|T| \kappa p / 3] .
$$

The probability that (13) fails for some $\kappa, S, T$ is thus at most

$$
n^{2} \sum_{t>0} \exp [(2-K / 3) t \log n]
$$

(where the $n^{2}$ covers choices for $s, \kappa \in[n]$ ), which is $o(1)$ if $K>12$.

Proposition 3.5. There is a $K$ such that w.h.p. $|T(\Pi)|<K p^{-1}$ for every balanced cut $\Pi$.

Proof. The number of possibilities for $\Pi=(A, B)$ and a $T \subseteq A$ of size $t:=\lceil K / p\rceil$ is less than $\exp _{2}\left[n+t \log _{2} n\right]$, while for such a $\Pi$ and $T$,

$$
\operatorname{Pr}(T(\Pi) \supseteq T)<\operatorname{Pr}(|\nabla(T, B)|<(1-\varepsilon) \operatorname{tnp} / 2)<\exp [-c t n p],
$$

with $c \approx \varepsilon^{2} / 4$ (using $\left.|B|>(1-\eta) n / 2\right)$. The proposition follows, e.g. with $K=4 \varepsilon^{-2}$. 
Proposition 3.6. There is a $K$ such that w.h.p. for every cut $\Pi=(A, B)$ and $x \in A \backslash X(\Pi)$,

$$
d_{Q_{e}(\Pi)}(x)<K / p
$$

Proof. By Proposition 3.2 it's enough to show that w.h.p. (15) holds whenever (say) $d(x) \leq(1+\varepsilon) n p$. Noting that a violation at $x$ (and some $\Pi$ ) implies that there are disjoint $S \subseteq V$ and $T \subseteq N(x)$ with $|T| \geq t:=(1-2 \varepsilon) n p / 4$, $|S|=s:=\lceil K / p\rceil$ and $|\nabla(S, T)|<\frac{\alpha}{1-2 \varepsilon} s|T| p=:(1-\zeta) s|T| p$, we find that the probability of such a violation with $d(x) \leq(1+\varepsilon) n p$ is at most

$$
n^{2} 2^{(1+\varepsilon) n p}\left(\begin{array}{l}
n \\
s
\end{array}\right) \exp \left[-\zeta^{2} s t p / 2\right],
$$

which is $o(1)$ for sufficiently large $K$ (e.g. $K=5000$ is enough).

\section{Proof of Lemma 2.2}

We will show that the "w.h.p." statement in Lemma 2.2 holds whenever we have the conclusions of Propositions 3.2 3.5. so we assume in this section that these conclusions hold for $K$, which we take to be the largest of the $K$ 's appearing in these propositions (so $K \approx 4 \varepsilon^{-2}$, which is what's needed in Propositions 3.3 and 3.5).

To keep the notation simple, we set, for a given $\Pi=(A, B)$ and $F$,

$$
I=F[A], J=F[A, B], L=G[A, B] \backslash J,
$$

and write, e.g., $I(x)$ for the set of edges of $I$ containing $x$.

We may assume that, given $\Pi, F$ maximizes $\varphi(F, \Pi)$ subject to the conditions of the lemma. Notice that this implies

$$
d_{I}(x) \geq d_{L}(x) / 2 \quad \forall x \in A,
$$

since if $x$ violates (16) then $F^{\prime}:=(F \backslash I(x)) \cup L(x)$ satisfies the conditions of the lemma (using $F[B]=\emptyset$ to say $F^{\prime}$ is triangle-free) and has $\varphi\left(F^{\prime}, \Pi\right)>$ $\varphi(F, \Pi)$. We will actually show that if (16) is added to our other assumptions then $I=\emptyset$, whence $F \subset \Pi$ and $\varphi(F, \Pi)=|F|<|\Pi|$; so we now assume (16).

Set $T=T(\Pi) \backslash X(\Pi), S=\left\{x \in A \backslash T: d_{I}(x)>\varepsilon n p\right\}, R=A \backslash(S \cup T)$, $T_{1}=\left\{x \in T: d_{I}(x)>\varepsilon n p\right\}$ and $T_{2}=T \backslash T_{1}$. Let

$$
M=|\{(x, y, z): x y \in I, x z \in L, y z \in G\}| .
$$


(Note $x y \in I \Rightarrow x, y \in A$ and then $x z \in L \Rightarrow z \in B$.) Since $F$ is trianglefree, we have

$$
\sum_{x \in A}\left|\nabla\left(N_{I}(x), N_{L}(x)\right)\right|=M \geq \sum_{x \in A}\left|\nabla\left(N_{I}(x), N_{J}(x)\right)\right| .
$$

So if we set $g(x)=\left|\nabla\left(N_{I}(x), N_{L}(x)\right)\right|$ and $f(x)=\left|\nabla\left(N_{I}(x), N_{J}(x)\right)\right|$ (for $x \in A$ ), then (17) says

$$
\sum_{x \in A}(g(x)-f(x)) \geq 0
$$

whereas we'll show

$$
\sum_{x \in A}(g(x)-f(x))<0 \text { unless } I=\emptyset .
$$

Proof. We first assert that

$$
g(x)-f(x)< \begin{cases}(1+4 \varepsilon) d_{I}(x) n p^{2} / 3 & \text { if } x \in S \\ (1+4 \varepsilon) d_{I}(x) n p^{2} / 6 & \text { if } x \in T_{1}\end{cases}
$$

To see this, rewrite

$$
g(x)-f(x)=\left|\nabla\left(N_{I}(x), N_{B}(x)\right)\right|-2\left|\nabla\left(N_{I}(x), N_{J}(x)\right)\right| .
$$

For $x \in S \cup T_{1}$, (11) (with (3)) gives $\left|\nabla\left(N_{I}(x), N_{B}(x)\right)\right|<(1+\varepsilon) p d_{I}(x) d_{B}(x)$ and $\left|\nabla\left(N_{I}(x), N_{J}(x)\right)\right|>(1-\varepsilon) p d_{I}(x) d_{J}(x)$, while

$$
d_{J}(x) \geq d_{B}(x) / 3
$$

for any $x \in A$ (since $d_{L}(x)+d_{J}(x)=d_{B}(x)$ and, according to (16) and (3), $d_{L}(x) \leq 2 d_{I}(x) \leq 2 d_{J}(x)$ ). Inserting these bounds in (20) and using (quite unnecessarily) $d_{B}(x) \leq d(x)-d_{I}(x)<(1+o(1)-\varepsilon) n p$ (see (10)) gives (19).

We next consider $x \in R \cup T_{2}$, and rewrite

$$
g(x)-f(x)=2\left|\nabla\left(N_{I}(x), N_{L}(x)\right)\right|-\left|\nabla\left(N_{I}(x), N_{B}(x)\right)\right| .
$$

We consider the two terms on the right separately, beginning with the second. Recalling that $I \cap Q(\Pi)=\emptyset$ and setting $d_{I}^{\prime}(x)=\left|N_{I}(x) \backslash T\right|$, $d_{I}^{\prime \prime}(x)=\left|N_{I}(x) \cap T\right|$, we have

$$
\left|\nabla\left(N_{I}(x), N_{B}(x)\right)\right| \geq \begin{cases}\alpha n p^{2}\left(d_{I}^{\prime}(x) / 2+d_{I}^{\prime \prime}(x) / 4\right) & \text { if } x \in R \\ \alpha n p^{2} d_{I}(x) / 8 & \text { if } x \in T_{2} .\end{cases}
$$


For the first term on the r.h.s. of (21) we have

$$
x \in R \cup T_{2} \Rightarrow\left|\nabla\left(N_{I}(x), N_{L}(x)\right)\right|<d_{I}(x) \cdot 4 \varepsilon n p^{2},
$$

using (13) and the fact that $x \in R \cup T_{2}$ implies $d_{L}(x) \leq 2 d_{I}(x) \leq 2 \varepsilon n p$. (In more detail: if $d_{L}(x) \leq d_{I}(x)$ then we use (13) with $S=N_{L}(x), T=N_{I}(x)$ and $\kappa=\varepsilon n p$; otherwise, we take $S=N_{I}(x), T=N_{L}(x)$ and $\kappa=\varepsilon n p$ to obtain the bound $d_{L}(x) \cdot 2 \varepsilon n p^{2} \leq d_{I}(x) \cdot 4 \varepsilon n p^{2}$.)

In particular, for $x \in T_{2}$ we have

$$
g(x)-f(x) \leq d_{I}(x) n p^{2}(8 \varepsilon-\alpha / 8) \leq 0 .
$$

Collecting the information from (19) and (21)-(24), we find that the sum in (18) is bounded above by

$$
n p^{2}\left[(1+4 \varepsilon)\left(\sum_{x \in S} \frac{d_{I}(x)}{3}+\sum_{x \in T_{1}} \frac{d_{I}(x)}{6}\right)+\sum_{x \in R}\left\{8 \varepsilon d_{I}(x)-\alpha\left(\frac{d_{I}^{\prime}(x)}{2}+\frac{d_{I}^{\prime \prime}(x)}{4}\right)\right\}\right] .
$$

So we just need to show that this is negative if $I \neq \emptyset$, which follows from

$$
\sum_{x \in R} d_{I}^{\prime}(x) \geq .9 \sum_{x \in S} d_{I}(x)
$$

and

$$
\sum_{x \in R} d_{I}^{\prime \prime}(x) \geq .9 \sum_{x \in T_{1}} d_{I}(x) \text { if (say) }\left|T_{1}\right| \geq \eta|S| .
$$

(If $\eta|S|>\left|T_{1}\right|$ then (26) is enough and we don't need the $d_{I}^{\prime \prime}$ terms in (25).) The proofs of (26) and (27) are similar and we just give the first.

Proof of (26). We may of course assume $S \neq \emptyset$. Since $\sum_{x \in S} d_{I}(x)=$ $\sum_{x \in R} d_{I}^{\prime}(x)+|I[S, T]|+2|I[S]|$, it's enough to show

$$
|\nabla(S, T)|+2|G[S]| \leq .1 \sum_{x \in S} d_{I}(x) .
$$

Notice that $|T|<K p^{-1}$ (see Proposition 3.5) and $|S|<(\eta / \varepsilon) n$ (by (2) and (11), the latter applied to $|\nabla(A, B)|)$. Combining these bounds with the conclusions of Proposition 3.4 (using $\kappa=(\eta / \varepsilon) n$ and $\kappa=K p^{-1} \log n$ respectively) gives $|G[S]| \leq|S|(\eta / \varepsilon) n p$ and

$$
|\nabla(S, T)| \leq 2 \max \{|S|,|T|\} K \log n \leq \begin{cases}2 K|S| \log n & \text { if }|S| \geq|T| \\ 2 K^{2} p^{-1} \log n & \text { if }|S|<|T|\end{cases}
$$

So, noting that $\sum_{x \in S} d_{I}(x)>|S| \varepsilon n p$ and that $2 K^{2} p^{-1} \log n$ is small relative to $\varepsilon n p$, we have (28). 


\section{$5 \quad$ Proof of Lemma 2.3}

For $\Pi=(A, B)$, let $\Pi^{*}=(A \backslash X(\Pi), B \cup X(\Pi))$. Propositions 3.2 and 3.5 imply that w.h.p.

$$
\begin{aligned}
\left|\Pi^{*}\right| & \geq|\Pi|+\sum_{x \in X(\Pi)}\left(d(x)-2 d_{B}(x)-|X(\Pi)|\right) \\
& \geq|\Pi|+|X(\Pi)| n p / 2 \quad \text { for every balanced } \Pi .
\end{aligned}
$$

If $Q$ and $\Pi$ are as in Lemma 2.3 and $\left|Q \cap Q_{v}(\Pi)\right|>(1+\varepsilon)^{-1}|Q|$ then, since $\left|Q \cap Q_{v}(\Pi)\right|<|X(\Pi)|(1-2 \varepsilon) n p / 4$ (by (5)), we have

$$
|Q|<\left(1-\varepsilon-2 \varepsilon^{2}\right)|X(\Pi)| n p / 4,
$$

and it follows that

$$
b(G) \geq\left|\Pi^{*}\right|>|\Pi|+2|Q|
$$

provided (29) holds. It is thus enough to show

Lemma 5.1. There is a $\delta>0$ such that w.h.p.

$$
b(G)>|\Pi|+|Q| \delta n p^{2}
$$

for every balanced cut $\Pi$ and $\emptyset \neq Q \subseteq Q_{e}(\Pi)$.

Proof. Set $\gamma=(1-2 \varepsilon) / 4, \alpha^{\prime}=\alpha /(1-2 \varepsilon)$, and let $\zeta$ be a positive constant satisfying

$$
\vartheta:=.9-2 \zeta / \gamma-\alpha^{\prime}>0 .
$$

By Proposition 3.6 it's enough to prove Lemma 5.1 when $d_{Q}(x)<K / p$ ( $K$ as in the proposition) for all $x \in A$. It's also easy to see that for any such $Q$ and $\tau \in[p, K]$, there is a bipartite $R \subseteq Q$ with

$$
d_{R}(x) \leq\lceil\tau / p\rceil \quad \forall x
$$

and $|R| \geq \frac{\tau}{2 K}|Q|$. (To see this, start with a bipartite $Q^{\prime} \subseteq Q$ with $\left|Q^{\prime}\right| \geq$ $|Q| / 2$. Assigning each edge of $Q^{\prime}$ weight $\tau / K$ gives total weight at each vertex at most $\lceil\tau / p\rceil$ (actually $\tau / p$ of course, but we want integers), and the Max-flow Min-cut Theorem then gives the desired $R$.) It thus suffices to prove Lemma 5.1 with $Q$ replaced by a bipartite $R \subseteq Q_{e}(\Pi)$ satisfying (30), where we set

$$
\tau=\max \{\zeta, p\}
$$


(We could of course just invoke [2] to handle large $p$, but it seems silly to avoid the few extra lines needed to deal with this easier case.)

For $X, Y$ disjoint subsets of $V, f: X \rightarrow\{k \in \mathbf{N}: k \geq \gamma n p\}$, and $R \subseteq\{\{x, y\}: x \in X, y \in Y\}$ satisfying (30) with $V(R)=X \cup Y$, denote by $E(R, X, Y, f)$ the event that there is a balanced cut $\Sigma=(A, B)$ with $R \subseteq Q_{e}(\Sigma)$,

$$
d_{B}(x)=f(x) \quad \forall x \in X
$$

and

$$
b(G)<|\Sigma|+\vartheta|R| \gamma n p^{2} .
$$

We will show

$$
\operatorname{Pr}(E(R, X, Y, f))<\exp \left[-.001|R| n p^{2}\right] .
$$

This is enough to prove Lemma 5.1 (with $R$ in place of $Q$ as discussed above), since the number of possibilities for $(R, X, Y, f)$ with $|R|=t$ is less than $\left(\begin{array}{c}n \\ 2 \\ t\end{array}\right) 2^{t} n^{t}<\exp [3 t \log n]$.

For the proof of (32) we think of choosing $G$ in stages:

(i) Choose all edges of $G$ except those in $\nabla(Y, V \backslash X)$.

(ii) Choose all remaining edges of $G$ except those belonging to the sets $\nabla\left(y, \cup_{x y \in R} N_{x}\right)$ for $y \in Y$.

(iii) Choose the remaining edges of $G$.

Let $G^{\prime}$ be the subgraph of $G$ consisting of the edges chosen in (i) and (ii), and let $(S, T)$ be a balanced cut of $G^{\prime}$ of maximum size among those satisfying

$$
X \cup Y \subseteq S \text { and } d_{T}(x)=f(x) \quad \forall x \in X .
$$

(Of course if there is no such cut then $E(R, X, Y, f)$ does not occur.) For each $y \in Y$ set $M(y)=\left(\cup_{x y \in R} N_{x}\right) \cap T$ and $F(y)=\sum_{x y \in R} f(x)$.

If $\Sigma=(A, B)$ and $R \subseteq Q_{e}(\Sigma)$, then

$$
d_{B}(x, y)<\alpha^{\prime} \min \left\{d_{B}(x), d_{B}(y)\right\} p \quad \forall\{x, y\} \in R .
$$

Our choice of $(S, T)$ gives

$$
\begin{aligned}
|\Sigma| & \leq\left|G^{\prime}[S, T]\right|+\sum_{y \in Y} \sum_{x y \in R} d_{B}(x, y) \\
& \leq\left|G^{\prime}[S, T]\right|+\sum_{y \in Y} \sum_{x y \in R} \alpha^{\prime} d_{B}(x) p \\
& =\left|G^{\prime}[S, T]\right|+\alpha^{\prime} p \sum_{y \in Y} F(y)
\end{aligned}
$$


for any balanced cut $\Sigma=(A, B)$ satisfying $X \cup Y \subseteq A$, (31) and (33), while

$$
b(G) \geq\left|G^{\prime}[S, T]\right|+\sum_{y \in Y}|\nabla(y, M(y))| .
$$

Suppose first that we are in the (main) case $p<\zeta$ (so $\tau=\zeta$ ). Then w.h.p. (depending only on $G^{\prime}$ ) we have

$$
\begin{aligned}
|M(y)| & \geq \sum_{x y \in R}\left[d_{T}(x)-\sum\left\{d_{T}\left(x, x^{\prime}\right): x \neq x^{\prime} \in N_{R}(y)\right\}\right] \\
& >(1-2 \zeta / \gamma) F(y)
\end{aligned}
$$

for each $y \in Y$, since for any $x \in X, d_{T}(x)=f(x) \geq \gamma n p$ and the inner sum in (36) is (w.h.p.) at most

$$
d_{R}(y) \max \left\{d\left(x, x^{\prime}\right): x, x^{\prime} \in V\right\}<\lceil\zeta / p\rceil(1+o(1)) n p^{2}<2 \zeta n p .
$$

So w.h.p. the sum in (35) has the distribution $\operatorname{Bin}(m, p)$ for some

$$
m>(1-2 \zeta / \gamma) \sum_{y \in Y} F(y) \geq(1-2 \zeta / \gamma)|R| \gamma n p,
$$

and exceeds $.9 m p$ with probability at least $1-e^{-.005 m p}>1-e^{-.001|R| n p^{2}}$; and whenever this happens, $b(G)$ exceeds the r.h.s. of (34) by at least

$$
\left(.9-2 \zeta / \gamma-\alpha^{\prime}\right) \sum_{y \in Y} F(y) p \geq \vartheta|R| \gamma n p^{2} .
$$

If $p \geq \zeta$, then $R$ is a matching and the inner sum in (36) is empty. So we have $m \geq|R| \gamma n p$ in (37), and in (38) can replace $.9-2 \zeta / \gamma-\alpha^{\prime}$ by $.9-\alpha^{\prime}$.

\section{Remarks}

We continue to write $G$ for $G_{n, p}$.

Of course the next goal is to prove Conjecture 1.2. At this writing we think we may know how to do this, but the argument envisioned goes well beyond present ideas and, if correct, will appear separately.

Theorem 2.1] It would be interesting to know whether Theorem 1.1 can be proved without Theorem 2.1. This is not to say that such a proof would 
necessarily help in proving Conjecture 1.2. but, consequences aside, it seems interesting to understand whether this relatively difficult ingredient is really needed, or is just a convenience. (The extension of Theorem 2.1 to larger $r$, suggested in [13, 14], was achieved by Conlon and Gowers in [4] and given a different proof, building on work of Schacht [19], by Samotij in [18.)

It has also seemed interesting to give an easier proof of Theorem 2.1. The original proof [14, 10] uses a sparse version of Szemerédi's Regularity Lemma [20] due to Kohayakawa [13] and Rödl (unpublished; see [13]), together with the triangle case of the "KLR Conjecture" of [14]. The proofs of [4] and [18] avoid these tools (they do use the "graph removal lemma" of [6] so for Theorem 2.1 itself the "triangle removal lemma" of Ruzsa and Szemerédi [17] - but not in an essential way [3]), but are rather difficult. Here we just mention that we do now know a reasonably simple proof of Theorem 2.1. This argument will appear in [5].

Homology. It's not too hard to show that (roughly speaking) if $p$ is as in Theorem [2.1, then w.h.p. every triangle-free $F \subseteq G$ with $|F| \geq|G| / 2$ has even intersection with most triangles of $G$. (This is essentially due to Frankl and Rödl [8, following an idea of Goodman [9]; see also [10, Sec.8.2].) Thus in thinking about a new proof of Theorem 2.1, we wondered whether some insight might be gained by understanding what happens when one replaces "most" by "all." This turns out not to be a new question:

Recall that the clique complex, $X(H)$, of a graph $H$ is the simplicial complex whose faces are the (vertex sets of) cliques of $H$. (For background on this and related topological notions, see for example [16, 11].) A precise conjecture, proposed by M. Kahle ([12]; see also [11]) and proved by him for $\Gamma=\mathbf{Q}$, is

Conjecture 6.1. Let $\Gamma$ be either $\mathbf{Z}$ or a field. For each positive integer $k$ and $\varepsilon>0$, if

$$
p>(1+\varepsilon)[(1+k / 2)(\log n / n)]^{1 /(k+1)},
$$

then w.h.p. $H_{k}(X(G), \Gamma)=0$ (where $H_{k}$ denotes kth homology group).

(For $k=0$ - with, of course, $H$ replaced by the reduced homology $\tilde{H}$ - this is a classical result of Erdős and Rényi [7] on connectivity of $G_{n, p}$.) The answer to the above question (on edge sets having even intersection with all triangles) is the case $k=1, \Gamma=\mathbf{Z}_{2}$ of Kahle's conjecture, and an easy consequence of the following precise statement (again taken from [5]), in which we take $Q$ to be the event that every edge of $G$ is in a triangle. 
Theorem 6.2. For any $p=p(n)$

$$
\operatorname{Pr}\left(Q \wedge H_{1}\left(X(G), \mathbf{Z}_{2}\right) \neq 0\right) \rightarrow 0 \quad(n \rightarrow \infty) .
$$

In other words, w.h.p. either $Q$ fails or the only subsets of $E(G)$ meeting each triangle an even number of times are the cuts. As it turned out, our original proof of this was based on Theorem 2.1. so was not all that helpful from the point of view mentioned above; but we do now know how to show it without using Theorem 2.1, and this was indeed helpful in suggesting a new proof for the latter.

\section{References}

[1] L. Babai, M. Simonovits and J. Spencer, Extremal subgraphs of random graphs, J. Graph Th. 14 (1990), 599-622.

[2] G. Brightwell, K. Panagiotou and A. Steger, Extremal subgraphs of random graphs, 2009, submitted. http://arxiv.org/abs/0908.3778 (Extended abstract: pp. 477-485 in SODA '07 (Proc. 18th ACM-SIAM Symp. Discrete Algorithms).

[3] D. Conlon, private communication.

[4] D. Conlon and T. Gowers, Combinatorial theorems in sparse random sets, arXiv:1011.4310v1 [math.CO].

[5] R. DeMarco, A. Hamm and J. Kahn, in preparation.

[6] P. Erdős, P. Frankl and V. Rödl, The asymptotic number of graphs not containing a fixed subgraph and a problem for hypergraphs having no exponent, Graphs Combin. 2 (1986), 112-121.

[7] P. Erdős and A. Rényi, On random graphs I, Publ. Math. Debrecen 6 (1959), 290-297.

[8] P. Frankl and V. Rödl, Large triangle-free subgraphs in graphs without $K_{4}$, Graphs and Combinatorics 2 (1986), 135-144.

[9] A.W. Goodman, On sets of acquaintances and strangers at any party, Amer. Math. Mon. 66 (1959), 778-783.

[10] S. Janson, T. Euczak and A. Ruciński, Random Graphs, Wiley, New York, 2000. 
[11] M. Kahle, Topology of random clique complexes, Disc. Math. 309 (2009), 1658-1671.

[12] M. Kahle, personal communication.

[13] Y. Kohayakawa, Szemerédi's regularity lemma for sparse graphs, pp. 216-230 in Foundations of Computational Mathematics (Rio de Janeiro, 1997), Springer, Berlin, 1997.

[14] Y. Kohayakawa, T. Łuczak and V. Rödl, On $K^{4}$-free subgraphs of random graphs, Combinatorica 17 (1997), 173-213.

[15] W. Mantel, Problem 28, Wiskundige Opgaven, 10:60-61, 1907.

[16] R. Meshulam, The clique complex and hypergraph matching, Combinatorica 21 (2001), 89-94.

[17] I. Ruzsa and E. Szemerédi, Triple systems with no six points carrying three triangles, pp. 939-945 in Combinatorics (Keszthely, 1976), Coll. Math. Soc. J. Bolyai 18, Vol. II.

[18] W. Samotij, Stability results for discrete random structures, arXiv:1111.6885 v1 [math.CO].

[19] M. Schacht, Extremal results for random discrete structures, submitted.

[20] E. Szemerédi, Regular Partitions of Graphs, pp. 399-401 in Problèmes combinatoires et théorie des graphes (Colloq. Internat. CNRS, Univ. Orsay, Orsay, 1976), Paris: Éditions du Centre National de la Recherche Scientifique (CNRS), 1978.

[21] P. Turán, Eine Extremalaufgabe aus der Graphentheorie, Mat. Fiz Lapook 48 (1941), 436-452.

Department of Mathematics

Rutgers University

Piscataway NJ 08854

rdemarco@math.rutgers.edu

jkahn@math.rutgers.edu 